\title{
El valor del cooperativismo en el posconflicto colombiano
}

The value of cooperativism for Colombian post-conflict

O valor do cooperativismo para o pós-conflito colombiano

\section{Lizandro Alfonso Cabrera ${ }^{1}$}

Recibido: 12 de agosto de 2020

Aprobado: 29 de octubre de 2020

Publicado: 12 de enero de 2021

Cómo citar este artículo:

Lizandro Alfonso Cabrera. El valor del cooperativismo en el posconflicto colombiano.

DIXI, vol. 23, $n^{\circ}$. 1, enero-junio 2021, 1-13.

DOI: https://doi.org/10.16925/2357-5891.2021.01.12

Artículo de reflexión. https://doi.org/10.16925/2357-5891.2021.01.12

1 Maestro de formación. Licenciado en Ciencias Sociales. Abogado. Especialista en Derecho Privado, Desarrollo Intelectual y Educación. Magister en Derecho. Par del Ministerio de Educación. Profesor de posgrados de la Universidad de la Amazonia. 


\section{Resumen}

Tema y alcance: demostrar el valor y el papel del cooperativismo como alternativa de una verdadera paz.

Metodología: se realiza un barrido inicial frente al estado real del proceso de paz, el avance de los acuerdos firmados en Colombia y el impacto que puede tener el cooperativismo en este proceso.

Características: la unión es el camino para mantener la esperanza de paz.

Hallazgos: la cooperativa es el camino de visibilizar esa Colombia que quiere progresar desde la provincia.

Conclusiones: el cooperativismo, entendido como la posibilidad de asociarse', de unir esfuerzos y sueños de manera organizada y comunal, se convierte en una herramienta para que esas personas que de verdad están decididas a cambiar de vida y a salir adelante puedan aportar en esta reconstrucción del país nacional ${ }^{2}$. Hay muchas maneras de que el cooperativismo apoye experiencias exitosas y mínimas posibilidades de fracasar. Pues la única, la primera y la mejor manera que tuvo, tiene y tendrá el hombre de generar revoluciones, entendidas como cambio, es la unión de fuerzas.

Palabras clave: acuerdo, cooperativismo, paz, posconflicto.

\section{Abstract}

Theme and scope: To demonstrate the value and role of cooperativism as an alternative for true peace.

Methodology: An initial sweep is made regarding the real state of the peace process, the progress of the agreements signed in Colombia and the impact that cooperativism can have in this process.

Characteristics: Union is the way to maintain the hope for peace.

Findings: The cooperative is the way to make visible that Colombia that wants to progress from the province. Conclusions: Cooperativism, understood as the possibility of associating, of joining efforts and dreams in an organized and communal way, becomes a tool for those people who are really determined to change their lives and move forward to contribute to this reconstruction of the national country. There are many ways for cooperativism to support successful experiences and minimal possibilities of failure. The only, the first and the best way that man has had, has and will have to generate revolutions, understood as change, is the union of forces.

Keywords: Agreement, cooperativism, peace, post-conflict.

\section{Resumo}

Tema e escopo: Demonstrar o valor e o papel do cooperativismo como alternativa para a verdadeira paz.

Metodologia: Faz-se uma varredura inicial sobre o estado do processo de paz, o andamento dos acordos firmados na Colômbia e o impacto que o cooperativismo pode ter neste processo.

Características: A união é a forma de manter a esperança pela paz.

Resultados: A cooperativa é a forma de tornar visível a Colômbia que quer progredir desde a província.

Conclusões: O cooperativismo, entendido como a possibilidade de associar, de somar esforços e sonhos de forma organizada e comunitária, torna-se um instrumento para aquelas pessoas que estão realmente decidi-

1 Nadia Eslinda Castillo Romero (Ed.). ECONOMÍA SOCIAL EN CONTEXTOS DE VIOLENCIA: MÉXICO Y COLOMBIA. Universidad Iberoamericana Puebla. (2018).

2 Humberto Serna Gómez y Mario Samuel Rodríguez Barrera. El sector solidario como alternativa para el desarrollo social e inclusivo en el postconflicto colombiano. COOPERATIVISMO \& DESARROLLO 107. Febrero 2016. https://doi.org/10.16925/co. v23i107.1250 
das a mudar de vida e seguir em frente para contribuir para esta reconstrução do país nacional. Existem muitas maneiras de o cooperativismo apoiar experiências de sucesso e possibilidades mínimas de fracasso. A única, primeira e melhor forma que o homem teve, tem e terá de gerar revoluções, entendidas como mudança, é a união de forças.

Palavras-chave: Acordo, cooperativismo, paz, pós-conflito.

\section{INTRODUCCIÓN}

La paz real y verdadera es mucho más que el final de la guerra ${ }^{3}$. En Colombia, hace más de treinta meses se firmó un acuerdo en el papel con el grupo armado de las Farc-EP ${ }^{4}$. Es cierto, las estadísticas muestran que los índices de violencia de los muertos puestos por el conflicto bajaron. Aquí se debe hacer un paréntesis para recordar que estas cifras nunca fueron tan reales como decían ser, pues los muertos del conflicto eran menos que los muertos puestos por la delincuencia común, por los accidentes de tránsito y por la violencia cotidiana. ${ }^{5}$

Hoy en día, se vive lo que se ha denominado como posconflicto o posacuerdo; pero sucede una situación muy interesante y es que el Estado colombiano, entendido como ese ente abstracto, como ese elemento intangible, que no existe pero que cuando uno piensa en él recurre a la Policía, recuerda al presidente de la República y lo relaciona con su gobierno. Y es así como las cooperativas están reemplazando al Estado en muchas de sus funciones sociales. Las cooperativas ofrecen créditos a muy bajo interés, capacitación en competencias laborales, capital semilla y las magníficas ruedas de negocios que aseguran relaciones de tipo comercial y financiero. El tejido social lo está construyendo el cooperativismo en Colombia.

\section{AVANCES DEL POSACUERDO}

A continuación, se presentan los avances de la implementación del acuerdo de paz. Junio 13 de 2017 | Avance en la dejación de armas de las Farc en zona veredal de La Elvira, Cauca.

3 Lizandro Cabrera-Suárez. La paz: mucho más que el final de la guerra. DIXı 23. Abril 2016. https://doi.org/10.16925/di.v18i23.1289

4 Héctor Olasolo y Jannluck Canosa Canosa. La responsabilidad del superior en el acuerdo de paz en Colombia a la luz del derecho internacional. PoLítICA CRIMINAL 25. Julio 2018. Pág. 444-500. http://dx.doi.org/10.4067/S0718-33992018000100444

5 Vicente Torrijos. CARTOgRAFía del CONFLICTO. PAUTAS INTERPRETATIVAS SOBRE LA EVOLUCIÓN DEL CONFLICTO IRREGULAR COLOMBIANO. Editorial Universidad del Rosario. (2015). 
Junio 8 de 2017 | La Misión de la onu en Colombia tiene en sus contenedores el $30 \%$ de las armas de las Farc.

Los quince embajadores de los países con asiento en el Consejo de Seguridad de Naciones Unidas viajaron a Colombia para observar de primera mano cómo avanza la implementación, y con una visita a la zona veredal de Vista Hermosa, le hicieron seguimiento al proceso de dejación de armas.

Al final de su visita, los embajadores ratificaron el apoyo del Consejo de Seguridad al acuerdo de paz. "Colombia ha iniciado el proceso de implementación y ya es irreversible, no hay vuelta atrás y el Consejo de Seguridad permanecerá a su lado", dijo el embajador británico Matthew Rycroft.

\section{Consejo de seguridad de la oNU visita Colombia y respalda implementación} Mayo:

Reunión con el propósito de asegurar el cumplimiento de los compromisos que prevé el Acuerdo Final, incluido lo relativo al D+180, y de proyectar la siguiente fase de implementación.

Mayo 29 de 2017 | Actualización del cronograma para la dejación de armas.

Abril:

En un acto con decenas de víctimas del conflicto, se firmaron tres decretos legislativos que dan vida al componente de verdad del capítulo de víctimas del Acuerdo:

- Comisión para el Esclarecimiento de la Verdad, la Convivencia y la No repetición.

- Unidad de Búsqueda de personas dadas por desaparecidas en el contexto y en razón del conflicto armado.

- Comité de Escogencia de carácter independiente e imparcial que elegirá a los altos funcionarios del Sistema Integral de Verdad, Justicia, Reparación y No repetición, incluyendo los comisionados de la Comisión de la Verdad, el director de la Unidad de Búsqueda de personas desaparecidas y los magistrados de la Jurisdicción Especial para la Paz

\section{Firma de decretos para el capítulo víctimas del acuerdo de paz}

Se instaló en la Cancillería el acompañamiento internacional a la implementación del Acuerdo, con la presencia de una decena de embajadas, organizaciones internacionales y agencias de las Naciones Unidas. 


\section{Instalación del acompañamiento internacional a la implementación}

El 18 de abril de 2017, comenzó a trabajar el comité de cinco personas que seleccionará, de manera autónoma e imparcial, a todos los magistrados y los jueces de la Jurisdicción Especial para la Paz.

Los cinco integrantes (Diego García-Sayán, Juan E. Méndez, Álvaro Gil-Robles, José Francisco Acuña y Claudia Vaca) fueron seleccionados, respectivamente, por el Secretario General de Naciones Unidas, el Centro Internacional para la Justicia Transicional (ICTJ), el Consejo Europeo de Derechos Humanos, la Corte Suprema de Justicia y los rectores de las universidades públicas.

Instalación del comité de selección de la Jurisdicción Especial para la Paz

El 26 de abril de 2017, el Congreso terminó la aprobación del Estatuto de la Oposición, que define las garantías para los partidos y movimientos políticos que se declaren en oposición al Gobierno.

Esta tarea, que aparece en el punto de participación política del Acuerdo y que da cumplimiento a una vieja tarea de la Constitución de 1991 que no se había ejecutado nunca, se cumplió tras seis meses de trabajo con todos los partidos y movimientos.

\section{Congreso aprueba Estatuto de la Oposición}

Se abrió el proceso para crear la Instancia Especial, que contribuirá a garantizar el enfoque de género en la implementación del Acuerdo Final.

Estará integrada por cuatro mujeres en representación de las organizaciones de mujeres a nivel territorial, dos mujeres por las organizaciones de mujeres a nivel nacional y una mujer en representación de las organizaciones LGBTI.

\section{Se abre convocatoria para instancia de mujeres}

Tras tres meses de trabajo, la Misión Electoral Especial entregó el informe en el que esboza sus recomendaciones sobre los ajustes normativos e institucionales necesarios para garantizar la modernización y la transparencia del sistema electoral colombiano, con el ánimo de que puedan ser discutidas por todos los partidos políticos y actores de la sociedad civil.

\section{Misión Electoral entrega su informe con propuestas electorales}

El Congreso aprobó la reincorporación política de las Farc, una de las piedras angulares de todo el acuerdo de paz, porque permite la transformación de una organización armada en una que hará política una vez haya dejado las armas.

6 Ana-María Fajardo-Fajardo. La JEP en 2017: un paso adelante y tres atrás. Working Paper. 2020. https://scholar.google.com/citations?user=gcqQvF8AAAAJ\&hl=es 


\section{Congreso aprueba la reincorporación política de las Farc}

El 1 de marzo, día D+90 en el calendario del fin del conflicto, comienza la dejación de armas por parte de las Farc.

La entrega del armamento de las Farc, desarrollado en tres fases hasta llegar al $100 \%$, se hace a los observadores de Naciones Unidas, organización que lidera el Componente Internacional del Mecanismo de Monitoreo y Verificación (MM\&V). Estas armas son almacenadas en un contenedor bajo el control exclusivo de la oNU.

\section{Comienza la dejación de armas}

Marzo:

El Congreso aprobó el acto legislativo que crea todo el Sistema Integral de Verdad, Justicia, Reparación y No Repetición, centro del punto sobre víctimas del Acuerdo. Ese acto crea también la Jurisdicción Especial para la Paz, que es el componente judicial del sistema.

Como dijo el alto comisionado Sergio Jaramillo, "la aprobación de la Jurisdicción Especial para la Paz es la base constitucional del sistema integral para las víctimas".

\section{Congreso aprueba la creación de la Jurisdicción Especial para la Paz}

La Comisión de Seguimiento, Impulso y Verificación a la Implementación (CSIVI) eligió a las universidades que designarán al grupo de tres expertos que, de acuerdo con la propuesta que hicieron líderes del 'No', presentará recomendaciones sobre reformas que permitan: regularizar los derechos de propiedad de los propietarios, ocupantes y poseedores de buena fe (siempre que no haya despojo o mala fe); garantizar la función social y ecológica de la propiedad; promover el uso productivo de la tierra; y facilitar el acceso a la tierra a quienes no la tienen.

Estos expertos serán escogidos por los rectores de la Universidad de los Andes, la Universidad Eafit y la Universidad Externado.

\section{Se conforma grupo de expertos en tierras}

El Gobierno nacional y las Farc, en el marco de la CSIVI, designarán el 30 de marzo de 2017 como notables del Componente Internacional de Verificación al expresidente español Felipe González y al expresidente uruguayo José Alberto Mujica Cordano.

7 Juliana Tonche y Camilo Eduardo Umaña. Sistema Integral de Verdad, Justicia, Reparación y No Repetición: un acuerdo de justicia ¿restaurativa? REVISTA DERECHO DEL ESTADO 38. Junio 2017. Pág. 223-241. https://doi.org/10.18601/01229893.n38.09 


\section{Se designan notables del componente internacional de verificación}

Los equipos de la Registraduría Nacional realizaron la cedulación de los integrantes de las Farc en las zonas veredales, como parte del proceso de reincorporación que aparece en el punto del fin del conflicto.

\section{Cedulación de integrantes de las Farc}

Durante este mes, las cinco instituciones designadas por el acuerdo de paz para escoger al Comité de Selección de magistrados y jueces de la Jurisdicción Especial para la Paz hicieron públicos a sus elegidos.

Es así como:

- Naciones Unidas seleccionó a Diego García-Sayán, excanciller peruano y exmagistrado de la Corte Interamericana de Derechos Humanos.

- El Centro Internacional para la Justicia Transicional (ICTJ) seleccionó a Juan E. Méndez, exrelator de Naciones Unidas sobre la Tortura y director emérito del ICTJ.

- El Consejo Europeo de Derechos Humanos seleccionó a Álvaro Gil-Robles, excomisario europeo de Derechos Humanos y exdefensor del Pueblo de España.

- La Corte Suprema de Justicia seleccionó a José Francisco Acuña, magistrado de la Sala Penal de la Corte Suprema.

- El Sistema Universitario Estatal, integrado por los rectores de todas las universidades públicas de Colombia, seleccionó a Claudia Vaca, profesora de la Universidad Nacional.

\section{Se escoge Comisión de Selección de magistrados de la Jurisdicción Especial} para la Paz

\section{Febrero:}

A lo largo de diez días, llegan 6934 integrantes de las Farc a las veintiséis zonas veredales en todo el país, donde realizarán el proceso de dejación de armas.

Ese gigantesco operativo se dio tras 36 desplazamientos físicos que involucran un total de 1980 vehículos, cien lanchas, diez tractores y veinte ambulancias.

\section{Se concentran 6934 integrantes de las Farc}

Se creó esta comisión que aparece en el punto de fin del conflicto; tiene como objetivo hacer seguimiento y desmantelar las organizaciones criminales que amenacen a líderes sociales y a la implementación del acuerdo de paz. 
En ella tienen asiento: el presidente de la república, el ministro del Interior, el ministro de Defensa, el ministro de Justicia, el fiscal general de la Nación, el defensor del Pueblo, el procurador general de la Nación, el director de la Unidad Especial de Investigación para el Desmantelamiento de las Organizaciones Criminales de la Fiscalía, el comandante de las Fuerzas Militares, el director general de la Policía Nacional y cinco representantes de la sociedad civil.

\section{Se instala la Comisión Nacional de Garantías de Seguridad}

Tras un periodo de seis meses de trabajo con todos los partidos y movimientos políticos, el Gobierno presentó ante el Congreso el proyecto de ley del Estatuto de la Oposición, que define los lineamientos del estatuto de garantías para quienes se declaren en oposición.

Esta tarea, que aparece en el punto de participación política del Acuerdo, da cumplimiento a una vieja tarea de la Constitución de 1991 que no se había realizado hasta ahora.

\section{Se presenta Estatuto de la Oposición al Congreso}

El Congreso aprobó, de acuerdo con el punto de fin del conflicto, la participación de seis personas del movimiento ciudadano Voces de Paz durante el trámite de los proyectos de ley de implementación del Acuerdo Final.

Estos seis voceros, tras la modificación a la Ley Quinta, tendrán voz pero no voto en las discusiones que se den en el Senado y en la Cámara de Representantes. Ellos son Imelda Daza, Jairo Estrada, Jairo Rivera, Francisco Tolosa, Judith Maldonado y Pablo Cruz.

Congreso aprueba la participación de seis personas del movimiento ciudadano Voces de Paz

Enero:

Se instaló la Misión Electoral Especial, cuyo fin es presentar recomendaciones sobre los ajustes normativos e institucionales necesarios para garantizar la modernización y la transparencia del sistema electoral colombiano.

La selección autónoma e independiente de los expertos fue delegada a cuatro importantes organizaciones: el Centro Carter, el Instituto Holandés para la Democracia Multipartidaria (NIMD), el Departamento de Ciencia Política de la Universidad Nacional y el Departamento de Ciencia Política de la Universidad de los Andes. Al cabo de cuatro meses y tras un proceso participativo con todos los partidos, los movimientos y las agrupaciones políticas, ellos presentarán su informe de recomendaciones. 
Sus integrantes son:

- Alejandra Barrios, directora de la Misión de Observación Electoral (MOE).

- $\quad$ Elisabeth Ungar, directora de Transparencia por Colombia.

- Alberto Yepes, magistrado del Consejo de Estado.

- Jorge Enrique Guzmán, profesor de la Universidad Javeriana.

- Juan Carlos Rodríguez-Raga, profesor de la Universidad de los Andes.

- Salvador Romero, director del Centro de Asesorías y Promoción Electoral del Instituto Interamericano de Derechos Humanos (CAPEL-IIHD).

\section{Se crea la Misión Electoral Especial}

Se instala la CSIVI, que aparece en el punto sobre implementación y verificación, para monitorear la puesta en marcha del acuerdo de paz.

En ella tienen asiento el alto comisionado Sergio Jaramillo, el consejero para el Posconflicto Rafael Pardo y el ministro del Interior Juan Fernando Cristo, además de las Farc.

\section{Se crea la Comisión de Seguimiento (csıvı)}

Diciembre:

El Congreso aprobó la Ley de Amnistía, que establece las condiciones para que (en el marco de la dejación de armas) los integrantes de las Farc que no hayan cometido crímenes graves o representativos soliciten la amnistía a la Jurisdicción Especial para la Paz.

Tras su paso por el Congreso, la ley está actualmente en revisión en la Corte Constitucional.

\section{Se aprueba Ley de Amnistía}

Se creó esta comisión que aparece en el punto sobre fin del conflicto y que tiene como misión definir las actividades, establecer el cronograma y hacerle seguimiento al proceso de reincorporación de los miembros de las Farc a la vida civil.

En ella, tienen asiento la ministra del Trabajo Clara López y el director de la Agencia Colombiana para la Reintegración Joshua Mitrotti, además de dos miembros de las Farc.

\section{Se instala el Consejo Nacional de Reincorporación}

Comenzó la puesta en marcha de las veintiséis zonas veredales y puntos transitorios de normalización, que acogerán a los integrantes de las Farc durante el periodo de dejación de las armas y el inicio de su reincorporación a la vida civil. 


\section{Puesta en marcha de las zonas veredales}

Comienza el cronograma de 180 días del punto del fin del conflicto, que fija los procedimientos mediante los cuales las Farc se concentrarán en las zonas veredales, dejarán la totalidad de sus armas e iniciarán su reincorporación a la vida civil.

\section{Día D+1}

Noviembre:

El 24 de noviembre de 2017, se firmó el Nuevo Acuerdo de Paz, que incorporó8 como cambios, ajustes y precisiones la mayor parte de los comentarios de los líderes del 'No'.

Posteriormente, el Acuerdo fue refrendado por el Congreso, por 70 votos a 0 en el Senado y 105 votos a 0 en la Cámara de Representantes.

Todo lo anterior demuestra el interés de ambas partes en materializar el Acuerdo. El ensayo trabaja el posacuerdo.

El Estado no ha hecho presencia donde antes no lo hacía. Colombia se acostumbró a que en más del 50 \% de los 1103 municipios, es decir, en cerca de 500 municipios el orden y la ley fueron impuestos por los actores armados. Cuando salieron las Farc, estos territorios quedaron 'libres' y fueron ocupados por otras fuerzas del mal, por la delincuencia común, por disidencias de las Farc o por otros grupos guerrilleros. El Estado no recuperó el territorio nacional.

Los excombatientes que no habían aprendido a vivir en comunidad y en una sociedad civil deambulaban por determinadas zonas a plomo y garrote, y eran extraños en su propio país. Las zonas de reclusión o las zonas veredales (en total veintitrés zonas veredales y ocho campamentos) casi siempre las ubicaron en espacios diferentes a los que ellos conocían. Las zonas de ubicación tendrán inicialmente una vigencia de 180 días, al cabo de los cuales la guerrilla deberá haber entregado hasta su última arma. Uno de los temas de mayor discusión en torno a los diálogos de paz entre el Gobierno y las Farc en Cuba fue el de las zonas de concentración donde se ubicarán los guerrilleros tras la suscripción del Acuerdo entre las partes, así como las normas que regirán en dichos lugares y el proceso para poner en práctica la dejación de armas.

Los enigmas respecto a estos asuntos quedaron despejados cuando el vocero del Gobierno cubano, Rodolfo Benítez, leyó el Acuerdo denominado "para el fin del conflicto". Según dicho documento, firmado en la misma ceremonia por el presidente Juan Manuel Santos y el máximo jefe de las Farc, Rodrigo Londoño, alias 'Timoleón

8 Jorge Eliécer Rojas-Naranjo. Postacuerdo y gestión territorial en Colombia. REVISTA BITÁCORA URBANO TERRITORIAL 2. Julio-diciembre 2016. Pág. 135-146. https://www. redalyc.org/pdf/748/74846551017.pdf 
Jiménez' y alias 'Timochenko', los miembros de la guerrilla se ubicarán en veintitrés zonas veredales y ocho campamentos, en donde habrá condiciones especiales. Por ejemplo, el tránsito de los subversivos a estas zonas se hará con el acompañamiento de miembros de la Fuerza Pública que garantizarán la seguridad.

La creación de dichas zonas fue clave porque se trata de los lugares hacia los cuales se deben desplazar los guerrilleros para concentrarse durante los meses previos a su desmovilización. Resultaría muy difícil que un grupo guerrillero ubicado en La Guajira, por ejemplo, tuviese que desplazarse hasta el Cauca o hasta Bogotá para dejar sus armas. Lo lógico es que en aquellas zonas del país próximas a dos o más frentes de las Farc se cree una zona para tal fin. En principio, las Farc querían más de ochenta zonas de concentración y el Gobierno les ofrecía nueve. Con el paso de la negociación, se definieron veintirés zonas veredales y ocho campamentos, es decir, las dos partes cedieron.

En las zonas de concentración, no se usarán uniformes. El Gobierno y las Farc acordaron un mecanismo de monitoreo tripartito entre la Fuerza Pública, las Farc y miembros de las Naciones Unidas, monitoreado por la comunidad internacional9 .

Cambiar el imaginario del común implicaba no solo el de los guerrilleros; sino que además la sociedad civil los aceptara y que el perdón fuera casi inmediato. Pero eso no fue así. Sucedió que la gente se quedó en las zonas veredales olvidada por el Estado, con promesas incumplidas, y proyectos y programas a medio desarrollar. Algunos comenzaron a recibir algún apoyo. Hoy en día, se encuentran en procesos productivos y de reintegro social los excombatientes que cambiaron sus actividades ilícitas por economías terciarias como agricultura de bajo nivel y ganadería.

El trabajo independiente e individual fue el abrebocas de la esquizofrenia del desengaño. Muchos regresaron al monte ${ }^{10}$.

\section{CONCLUSIONES}

El cooperativismo, entendido como la posibilidad de asociarse ${ }^{11}$, de unir esfuerzos y sueños de manera organizada y comunal, se convierte en una herramienta para que

9 https://cutt.ly/7WZePiKI

10 Remedios Pitre-Redondo, Diego Cardona-Arbeláez y Hugo Hernández-Palma. Proyección del emprendimiento indígena como mecanismo de competitividad en el postconflicto colombiano. REVISTA DE INVESTIGACIÓN, DESARROLLO E INNOVACIÓN 2. Enero-junio 2017. Pág. 231-240. https://doi.org/10.19053/20278306.v7.n2.2017.6068

11 Nadia Eslinda Castillo Romero, supra, nota 2. 
esas personas que de verdad tienen la decisión de cambiar de vida y de salir adelante puedan aportar en esta reconstrucción del país nacional12.

Hay muchas maneras de que el cooperativismo apoye experiencias exitosas y mínimas posibilidades de fracasar. Pues la única, la primera y la mejor manera que tuvo, tiene y tendrá el hombre de generar revoluciones, entendidas como cambio, es la unión de fuerzas. La cooperativa es el camino de visibilizar a esa Colombia que quiere progresar desde la provincia.

Un excombatiente es un ser humano. Tiene un saber hacer. Es el hijo de alguna madre, sus hijos lo aman y lucha por volver a ser un ciudadano del común. El camino se inicia con el apoyo, con las capacitaciones, con el capital semilla y con los negocios. Todo eso es el cooperativismo en el posconflicto.

\section{Recomendaciones}

1. Las cooperativas tienen un papel fundamental en la economía social, ya que son agentes de desarrollo económico con impacto social. Su presencia está en todos los sectores económicos. Debido a la incapacidad del mercado, el sector público y el sector privado para satisfacer las necesidades de la sociedad y de ciertos grupos vulnerables, se fomenta la creación de nuevas formas de organización que componen a la economía social, también conocida como el "tercer sector".

2. Las cooperativas son un modelo de empresa en el cual se integran los objetivos económicos, empresariales y sociales, con el fin de alcanzar un crecimiento basado en empleo, equidad social e igualdad.

3. Las cooperativas son necesarias para impulsar el desarrollo rural. Se caracterizan por sus principios de solidaridad, por su compromiso con las personas y con la generación de empleo, por ser democráticos y contribuir a la estabilidad de los mercados económicos, y permiten que las personas contribuyan activamente a la viabilidad económica de las zonas rurales. En Colombia, se ha mostrado una gran actividad por parte de estas unidades económicas, ya que buscan reactivar la economía con base en los microcréditos. Con el tiempo, se han incrementado las cooperativas y han mostrado una consolidación en el mercado financiero. 


\section{REFERENCIAS}

